\title{
Tripartite interactions between two phase qubits and a resonant cavity
}

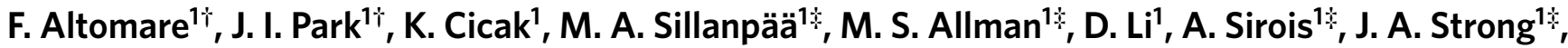 \\ J. D. Whittaker ${ }^{1 \star}$ and R. W. Simmonds ${ }^{1 \star}$
}

\begin{abstract}
Multipartite entanglement is essential for quantum computation $^{1}$ and communication ${ }^{2-4}$, and for fundamental tests of quantum mechanics ${ }^{5}$ and precision measurements ${ }^{6}$. It has been achieved with various forms of quantum bits (qubits), such as trapped ions ${ }^{7,8}$, photons ${ }^{9}$ and atoms passing through microwave cavities ${ }^{10}$. Quantum systems based on superconducting circuits, which are potentially more scalable, have been used to control pair-wise interactions of qubits ${ }^{11-16}$ and spectroscopic evidence for three-particle entanglement was observed ${ }^{17,18}$. Here, we report the demonstration of coherent interactions in the time domain for three directly coupled superconducting quantum systems, two phase qubits and one resonant cavity. We provide evidence for the deterministic evolution from a simple product state, through a tripartite W state, into a (bipartite) Bell state. The cavity can be thought of as a multiphoton register or an entanglement bus, and arbitrary preparation of multiphoton states in this cavity using one of the qubits ${ }^{19}$ and subsequent interactions for entanglement distribution should allow for the deterministic creation of another class of entanglement, a GreenbergerHorne-Zeilinger state.
\end{abstract}

With the development of quantum information science ${ }^{1}$, entanglement of multiparticle systems has become a resource for a new information technology. In particular, three-particle or tripartite entanglement allows for teleportation ${ }^{2}$, secret sharing ${ }^{4}$ and dense coding ${ }^{20}$, with connections to cosmology ${ }^{21}$. Over the past decade, the development of exquisite control over quantum systems has led to various demonstrations of tripartite entanglement ${ }^{8-10}$. Genuine tripartite entanglement is delineated by two inequivalent classes of states ${ }^{22}$ : Greenberger-Horne-Zeilinger and W, where the W state involves only a single photon shared amongst three systems. Using multipartite entanglement in a solid-state-qubit system has only recently received theoretical attention ${ }^{23-25}$. Thus far in superconducting systems, bipartite entanglement has been verified by two-qubit quantum state tomography ${ }^{13}$ and used to carry out a quantum algorithm ${ }^{15}$. Spectroscopic evidence for three-particle entanglement was observed for two current-biased phase qubits coupled to a lumped element consisting of an inductor-capacitor circuit and a cavity as well as for transmon qubits ${ }^{17,18}$. In the experiments described below, we first verified the spectroscopic signature of three coupled systems. Next, we demonstrated coherent interactions. Frequency detuning of the third system was used to verify the proper change in the time evolution of two versus three coupled systems. Finally, we describe a free-evolution process as a means of deterministically preparing arbitrary single-photon tripartite entangled states and a corresponding visualization technique. We present evidence for the proper operation of this protocol by measuring the time-dependent behaviour of the two phase qubits. Here, entanglement is not verified directly, but the data are consistent with theoretical predictions. Proper execution of this protocol can prepare the system in a Bell or W state, as well as arbitrary entangled states.

In Fig. 1a, we show an optical micrograph of two qubits, qubit 1 and qubit 2, capacitively coupled to either end of an open-ended coplanar waveguide cavity with a half-wave resonant mode frequency of $\omega_{\mathrm{c}} / 2 \pi \approx 8.9 \mathrm{GHz}$. These cavities have shown coherent properties at the single-photon level ${ }^{14}$. Flux-biased phase qubits $^{26}$ can be thought of as anharmonic inductor-capacitor oscillators in which a single Josephson junction provides enough nonlinearity to address the two lowest oscillatory phase states $|g\rangle$ and $|e\rangle$. The energy-level separation $\hbar \omega_{j} \equiv E_{\mathrm{e}}-E_{g}$ can be independently tuned over a range $\sim 7-10 \mathrm{GHz}$ on the $j$ th qubit by use of inductively coupled flux bias coils. Another coil allows us to apply microwave pulses and fast bias shifts, also used for single-shot state measurement ${ }^{14}$. Independent state readout on the $j$ th qubit is accomplished by use of an inductively coupled d.c. superconducting quantum interference device. We describe this system using a two-qubit Jaynes-Cummings or Tavis-Cummings model $^{27}$. In a frame rotating at reference frequency $\omega_{\mu}$, we approximate the Hamiltonian of the system as

$$
H=\hbar \Delta_{\mathrm{c}} a^{\dagger} a+\sum_{j=1,2}\left[\hbar \Delta_{j} \sigma_{j}{ }^{+} \sigma_{j}^{-}+i \hbar g_{j}\left(\sigma_{j}^{+} a-a^{\dagger} \sigma_{j}^{-}\right)\right]
$$

where the mode operators $\sigma_{j}{ }^{ \pm}$and $a^{(\dagger)}$ refer to the qubits and the cavity, respectively, with corresponding detunings $\Delta_{j} / \hbar \equiv \omega_{j}-\omega_{\mu}$ and $\Delta_{\mathrm{c}} / \hbar \equiv \omega_{\mathrm{c}}-\omega_{\mu}$. Capacitive coupling $C_{\mathrm{c}}$ between the qubits and the cavity results in an effective coupling frequency of $2 g / 2 \pi \approx$ $\left(\omega_{\mathrm{c}} / 2 \pi\right) C_{\mathrm{c}} / \sqrt{C C_{\mathrm{J}}} \sim 90 \mathrm{MHz}$ for both qubits. The system exhibits decay rates of $\gamma_{1} / 2 \pi \sim 7 \mathrm{MHz}, \gamma_{2} / 2 \pi \sim 10 \mathrm{MHz}$ and $\kappa / 2 \pi \sim 1 \mathrm{MHz}$ for each qubit and the cavity, respectively. We denote the product of two-qubit/cavity states as $\left|\eta \eta^{\prime} n\right\rangle \equiv|\eta\rangle_{1} \otimes\left|\eta^{\prime}\right\rangle_{2} \otimes|n\rangle_{c}$, where $|\eta\rangle_{j}$ label the $j$ th qubit state $(|g\rangle$ or $|e\rangle)$ and $n$ labels the cavity Fock state.

The first signature of tripartite interactions is revealed by spectroscopic measurements ${ }^{17,18}$ as a function of the detuning $\Delta_{1, \mathrm{c}} / \hbar=\omega_{1}-\omega_{\mathrm{c}}$ of qubit 1 when qubit 2 and the cavity are resonant $\left(\omega_{2}=\omega_{c}\right)$. In the case of a single-qubit/cavity system, the JaynesCummings model predicts a single vacuum Rabi-mode splitting of the qubit state ${ }^{14}$. Here, the single-qubit states are split twice by the mutual interaction of all three systems, as shown in Fig. 1b. We can interpret this as being due to the coupling between the bare qubit 1 and the antisymmetric pair of maximally entangled Bell states between qubit 2 and the cavity. The two avoided crossings in the 

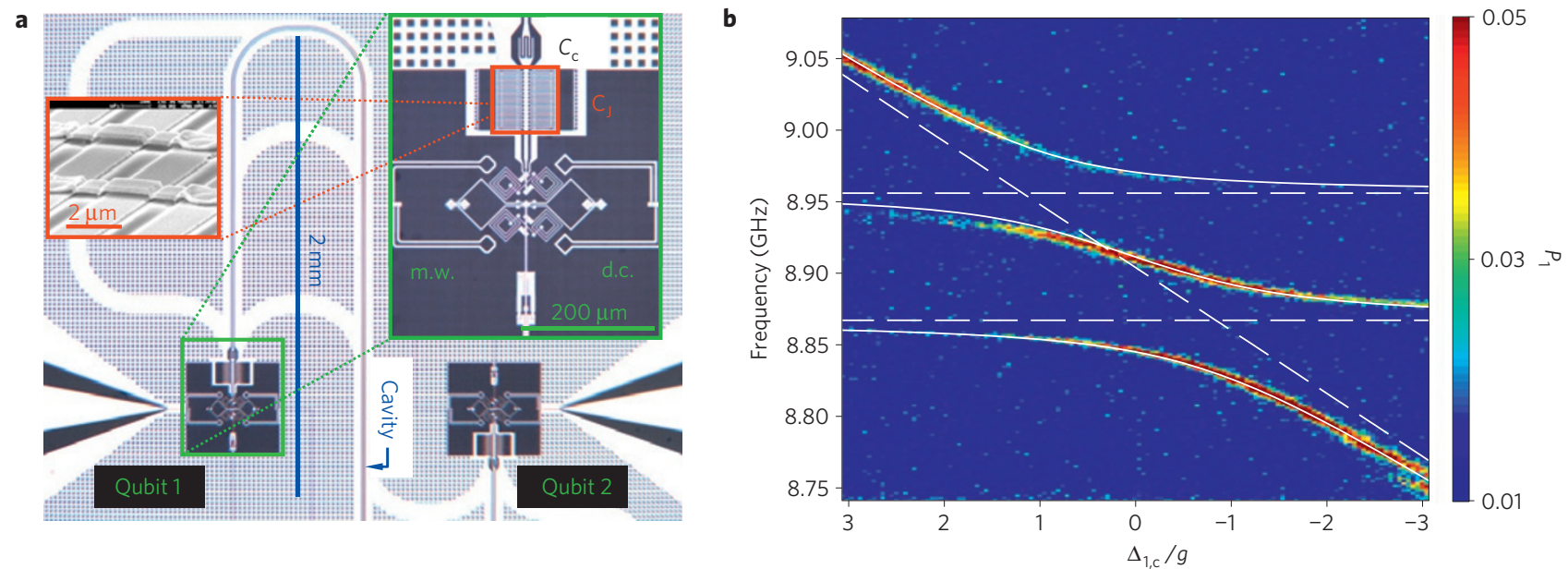

Figure 1 | Circuit and spectroscopy. a, Optical micrograph of the electrical circuit with two Josephson phase qubits (qubit 1 inset overlay right), each with loop inductance $\sim 700 \mathrm{pH}$ and critical current $\sim 0.91 \mu \mathrm{A}$ (junction areas $\sim 7 \mu \mathrm{m}^{2}$ ) shunted by use of interdigitated capacitors $\left(C_{j} \sim 0.7 \mathrm{pF}\right.$, including junction capacitance) with vacuum gap crossovers (inset overlay left), capacitively coupled $\left(C_{c} \sim 6.2 \mathrm{fF}\right.$ ) to a coplanar waveguide resonant cavity (of full length $\sim 7 \mathrm{~mm}$ ). The device was fabricated with standard optical lithography with $\mathrm{Al} / \mathrm{AlO}_{x} / \mathrm{Al}$ junctions on a sapphire substrate, with $\mathrm{SiO}{ }_{2}$ as an insulator surrounding the junctions. b. Microwave spectroscopy of qubit 1 as a function of detuning $\Delta_{1, \mathrm{c}}=\omega_{1}-\omega_{\mathrm{c}}$ with $\omega_{2}=\omega_{\mathrm{c}}$. $\Delta_{1, \mathrm{c}}$ is varied through the d.c. flux bias coils and qubit 1 is excited by microwaves applied through the m.w. (microwave) coil (seen in $\mathbf{a}$ ). The intensity colour scale represents the probability of qubit 1 tunnelling after the measure pulse. The dashed diagonal line shows the bare qubit 1 transition frequency. The dashed horizontal lines represent the energies of the two maximally entangled Bell states between qubit 2 and the cavity in the absence of coupling to qubit 1 .

spectrum occur along the qubit 1 detuning curve, symmetrically displaced about the tripartite resonance $\left(\omega_{1}=\omega_{2}=\omega_{\mathrm{c}}\right)$. These measured curves agree well with a full analysis of the two-qubit Jaynes-Cummings or Tavis-Cummings ${ }^{18}$ model.

With independent control over both qubits, we can easily explore a convenient state-space whereby a single photon of energy $\hbar \omega_{c}$ is shared by our tripartite system. Using a similar technique established for inducing coherent interactions between a single qubit and a cavity ${ }^{14}$, we investigate the evolution of vacuum Rabi oscillations between qubit 1 and the cavity as a function of the detuning $\Delta_{2, \mathrm{c}} / \hbar=\omega_{2}-\omega_{\mathrm{c}}$ of qubit 2 from the joint qubit 1-cavity system $\left(\omega_{1}=\omega_{\mathrm{c}}\right)$. For simplicity, we use the term 'photon' even when describing a single excitation in the qubit. We begin with both qubits in their ground state and qubit 1 far off-resonance from the cavity (see pulse diagram in Fig. 2a); then we excite qubit 1 with a photon using a $\pi$ pulse and bring it onto resonance with the cavity (using a shift pulse) for a given evolution time period $t_{\mathrm{e}}$ followed by simultaneous measurement of both qubits ${ }^{12}$. When qubit 2 is far enough off-resonant, the resultant vacuum Rabi oscillations are characterized by the frequency $\Omega_{0} \equiv 2 g$, as seen on either side of Fig. 2b,c. Here, the exchange between qubit 2 and the qubit-1-cavity system is energetically prohibited, so that qubit 1 undergoes basic vacuum Rabi oscillations with the cavity alone. When all three systems are on resonance, the photon appears to oscillate between the two qubits through the cavity. Beginning in qubit 1 , the photon 'spreads out' to the cavity, also becoming shared with qubit 2, then it moves completely to qubit 2 , eventually returning back to qubit 1 . In this antisymmetric mode (also see Fig. $2 \mathrm{f}$ ), the oscillation frequency is given by $\Omega_{\mathrm{a}}=\Omega_{0} / \sqrt{2}$. As the system evolves, the photon is never completely transferred to the cavity. There are times when the photon is entirely in qubit 1 or entirely in qubit 2, otherwise the system occupies a continuum of entangled states of both qubits and the cavity. By measuring the two qubits simultaneously ${ }^{12}$, we can extract the joint probabilities $P_{e g 0}$ and $P_{g e 0}$ for single-photon states $|e g 0\rangle$ and $|g e 0\rangle$, respectively. The experimental data agree well with the theoretical simulations (Fig. 2d-f) described in the Methods section.

The above experiment lends itself to a simple geometric description that can help us visualize the system dynamics. Using equation (1), we can identify the unitary evolution $U(t)=\mathrm{e}^{-\mathrm{i} H t / \hbar}$ of the system with a three-dimensional rotation $R_{\mathbf{n}}(\varphi)=\mathrm{e}^{-\mathrm{in} \cdot \mathbf{X} \varphi}$ about $\mathbf{n} \equiv\left(0, g_{2},-g_{1}\right) / \sqrt{g_{1}^{2}+g_{2}^{2}}$ with $\varphi=\sqrt{g_{1}^{2}+g_{2}^{2}} t$ and $\mathbf{X} \equiv\left(X_{1}, X_{2}, X_{3}\right)$. Here, $\left(X_{k}\right)_{i j}=-i \epsilon_{i j k}$ helps generate the rotation, and $\epsilon_{i j k}$ is the totally antisymmetric Levi-Civita tensor. Time evolution of the system then corresponds to orbits on a unit sphere azimuthal to the vector $\mathbf{n}$, where $(x, y, z) \Leftrightarrow(|g g 1\rangle,|\operatorname{eg} 0\rangle,|g e 0\rangle)$, as shown in Fig. 2g,h. By taking the amplitudes of the three coupled states as real, absorbing any overall phase into a redefinition of the states, we can construct a (unit) state vector analogous to that used for a single spin-1/2 system on the Bloch sphere. In this case, as the state vector precesses about $\mathbf{n}$ and away from any of the coordinate axes, entanglement evolves over time between all three systems. For the experiment described above, we start with an initial condition corresponding to the state $|\operatorname{eg} 0\rangle$. When qubit 2 is far off-resonance (Fig. $2 \mathrm{~g}$ ), the system precesses at $\Omega_{o}$ about $\mathbf{n}=(0,0,-1)$, showing simple vacuum Rabi oscillations between qubit 1 and the cavity involving the states $|\operatorname{eg} 0\rangle$ and $|g g 1\rangle$, generating bipartite entanglement. However, when all three systems are on-resonance $\left(\omega_{1}=\omega_{2}=\omega_{\mathrm{c}}\right), H=g_{2} X_{2}-g_{1} X_{3}=g\left(X_{2}-X_{3}\right)$, $\mathbf{n}=(0,1,-1) / \sqrt{2}$ and $\varphi=\sqrt{2} g t$, leading to a 'tripartite evolution'. Now the initial-state vector $|e g 0\rangle$ precesses about $\mathbf{n}$ so that the trajectory passes from the $\mid$ eg 0$\rangle$ axis into a region where the photon is shared with the cavity and then through the $-|g e 0\rangle$ axis (see Fig. 2h). The oscillations in the two qubits then follow the antisymmetric mode frequency $\Omega_{\mathrm{a}}$. In this case, the local operations are two- and three-particle vacuum Rabi oscillations that occur during the system's evolution. We can see that any arbitrary single-photon tripartite state can be created and subsequently transformed into any other state on the tripartite sphere, much like unitary operations and rotations on the Bloch sphere. Of particular interest is the fact that a specific initial state will follow a specific trajectory under tripartite evolution, transforming the amount of entanglement continuously. Below, we determine the conditions for directly demonstrating transformations between Bell and W states, starting from an initially pure state.

We begin with a single photon in qubit 1 or qubit 2 . As shown above, vacuum Rabi oscillations represent arbitrary rotations in the $|g g 1\rangle-|e g 0\rangle$ plane (between the cavity and qubit 1) or the $|g g 1\rangle-|g e 0\rangle$ plane (cavity and qubit 2). These two operations in 


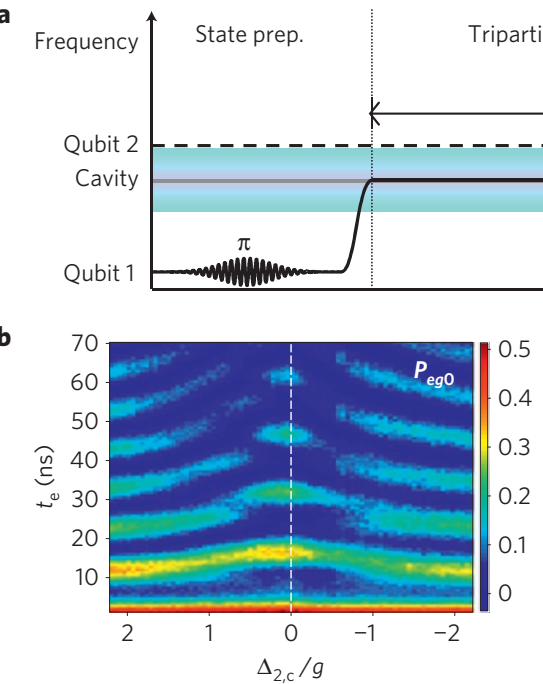

d

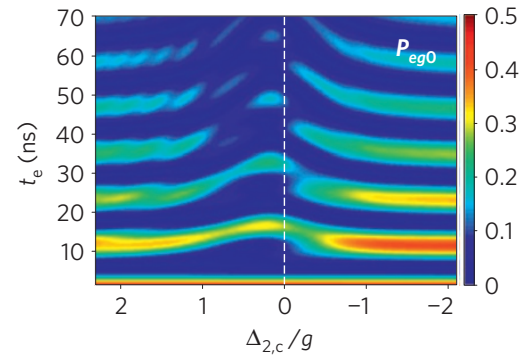

Tripartite evolution

t

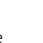

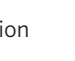
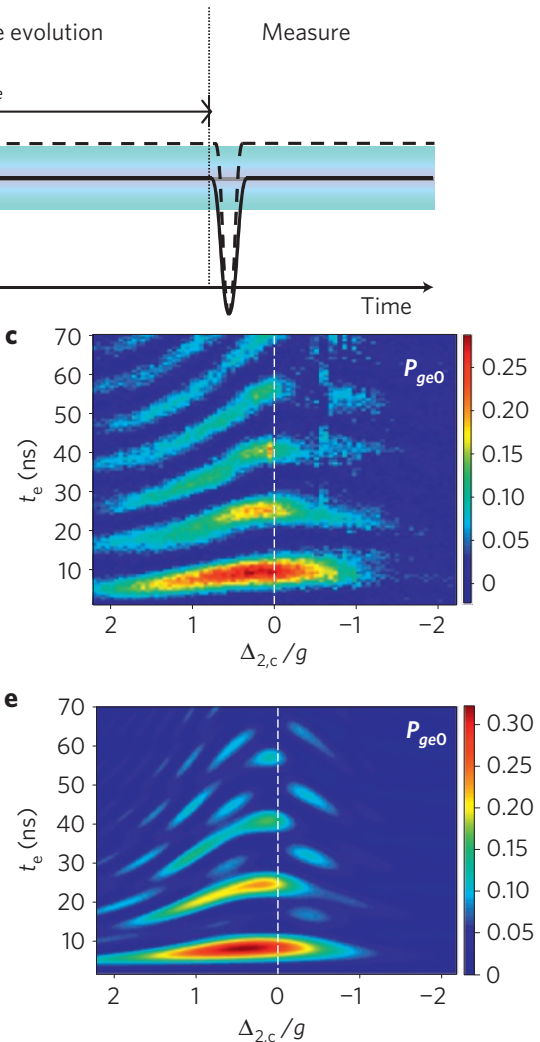

f

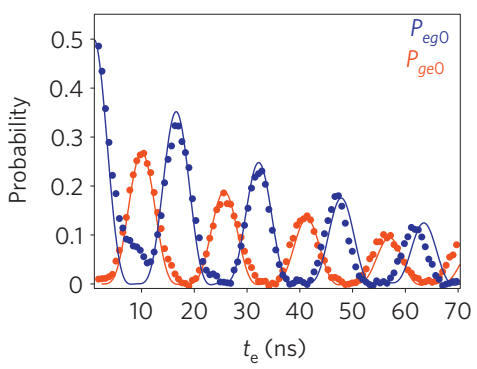

g

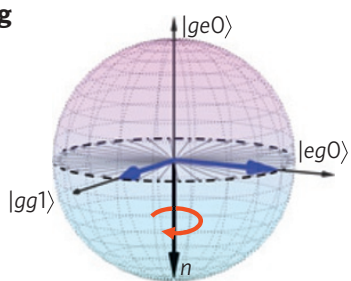

h

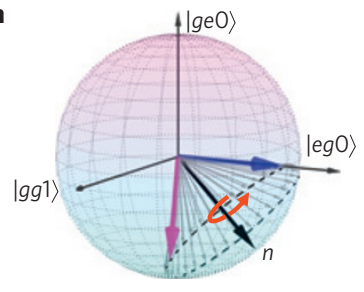

Figure 2 | Demonstration of basic tripartite interactions. a, Description for creating a photon in qubit 1 by use of a $\pi$ pulse, then shifting (solid line) onto resonance with the cavity and qubit 2 for various qubit 2 detunings (dashed line). After an evolution time period $t_{\mathrm{e}}$ the qubits are measured simultaneously ${ }^{12}$. b,c, Measured excited-state joint probabilities $P_{e g 0}$ and $P_{g e 0}$ for states $|e g 0\rangle$ and $|g e 0\rangle$, respectively, during tripartite interactions after qubit 1 has been excited by a $\pi$ pulse and shifted onto resonance with the cavity as a function of the detuning $\Delta_{2, c}=\omega_{2}-\omega_{c}$ of qubit 2 . d,e, Theoretical predictions including energy relaxation and the finite rise time of the shift pulse (see the Methods section). $\mathbf{f}$, Line cut of the on-resonance tripartite interactions with corresponding theoretical prediction (solid line). The red arrow in $\mathbf{g}, \mathbf{h}$ provides a visual cue to the circular trajectory of the tripartite vector. $\mathbf{g}$, Tripartite sphere representation during simple vacuum Rabi oscillations of qubit 1. $\mathbf{h}$, Tripartite sphere representation during the tripartite evolution from the initial state $|e g 0\rangle$.

succession allow us complete access to the $|e g 0\rangle-|g e 0\rangle$ plane, and, thus, the ability to prepare any initial state on the entire single-photon tripartite sphere. To generate Bell and $\mathrm{W}$ states, we can start with the photon in the cavity, $|g g 1\rangle$. Under tripartite evolution the system passes first through the $\mathrm{W}$ state, $|\mathrm{W}\rangle \equiv$ $(+|g g 1\rangle-|e g 0\rangle-|g e 0\rangle) / \sqrt{3}$, and then through the Bell state, $\mid$ Bell $\rangle \equiv-(|e g 0\rangle+|g e 0\rangle) / \sqrt{2}$, as the system vector rotates about the $n$ vector, $\mathbf{n}=(0,1,-1) / \sqrt{2}$ as shown in Fig. $3 b$. In total, the system will pass through two Bell states and four W states for one full revolution about $\mathbf{n}$. The frequency $\Omega_{\mathrm{s}}=\sqrt{2} \Omega_{0}$ of qubit oscillations follows from the definition of $\varphi$ and the arc traced out by the system trajectory. In this symmetric mode $\left(\Omega_{\mathrm{s}}=2 \Omega_{\mathrm{a}}\right)$ the cavity photon 'splits', having an equal probability for going to qubit 1 or qubit 2 , and subsequently returning completely to the cavity.

Experimentally, we sample a variety of initial states by allowing qubit 1 (which starts with the photon) to undergo vacuum Rabi oscillations with the cavity for a delay time period $t_{\mathrm{d}}$ before we bring qubit 2 into tripartite resonance. Figure $3 \mathrm{c}-\mathrm{e}$ shows a prediction for the unitary evolution of the system for nearly a continuum of values for $t_{\mathrm{d}}$. Here, the joint probabilities are $P_{g g 1}, P_{e g 0}$ and $P_{g e 0}$ for states $|g g 1\rangle,|e g 0\rangle$ and $|g e 0\rangle$, respectively. Notice that for $t_{\mathrm{d}}=2 \pi / \Omega_{0}$, the system will exhibit the antisymmetric mode (indicated along the dashed line) as described earlier. However, when $t_{\mathrm{d}}=\pi / \Omega_{0}$, we prepare (the initial state) $|g g 1\rangle$, allowing for a tripartite evolution of the symmetric mode. After a period of time $t_{\mathrm{e}}=\pi / 4 \Omega_{\mathrm{s}}$, the excited-state probability for both qubits is $1 / 3$ and the system is in the $|W\rangle$ state, with the photon equally distributed among the two qubits and cavity. After a period of time $t_{\mathrm{e}}=\pi / 2 \Omega_{\mathrm{s}}$, the excited-state probability for both qubits is $1 / 2$ and the system is in the bipartite |Bell $\rangle$ state. These points are indicated in Fig. 3c-e, with the first three states shown as vectors on the tripartite sphere in Fig. 3b. Here the simulations include energy relaxation and the finite rise time of the shift pulses (see the Methods section).

We simultaneously measure both qubits and observe the occupation probabilities of the two qubits over time as they evolve from a continuum of initial states, superposition states of qubit 1 and the cavity. Although possible, as explained later, we do not measure the cavity state directly. Figure $4 \mathrm{c}, \mathrm{d}$ shows extracted line cuts from Fig. 4a,b for two initial conditions (dashed lines) corresponding to the symmetric and antisymmetric modes. As can been seen from Fig. 3d,e, the theoretical predictions for the evolutions agree with the measurements. For the symmetric mode we find in-phase oscillations of the two qubits at $\Omega_{\mathrm{s}} \sim \sqrt{2} \Omega_{0}$, whereas for the antisymmetric mode, we find that the two qubits oscillate out of phase with each other with the antisymmetric mode frequency $\Omega_{\mathrm{a}} \sim \Omega_{0} / \sqrt{2}$, where $\Omega_{0}$ is the frequency of the vacuum Rabi oscillations that occur during the delay time period $t_{\mathrm{d}}$ (lower right-hand corner of Fig. $4 \mathrm{a}$ ). The measured frequencies agree within $\sim 15 \%$ of the ideal case, owing to the finite rise time of the shift pulses and some residual non-zero detuning of each qubit frequency. Theoretical simulations including these imperfections (solid lines) agree well with the data.

In the present experiment, we improved the previous design ${ }^{14,28}$ by reducing the qubit junction areas to reduce the number 


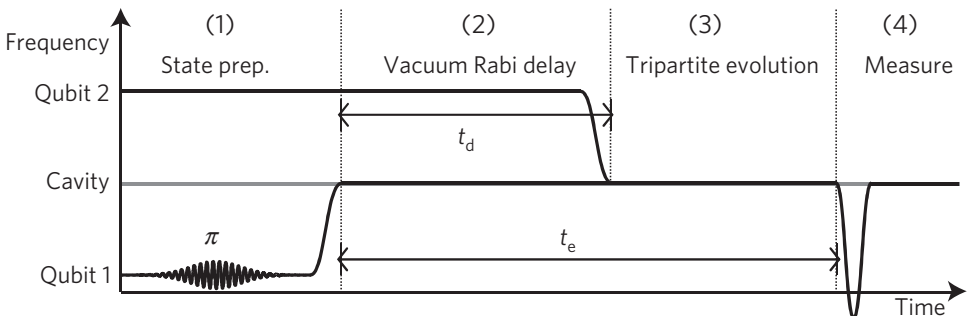

c
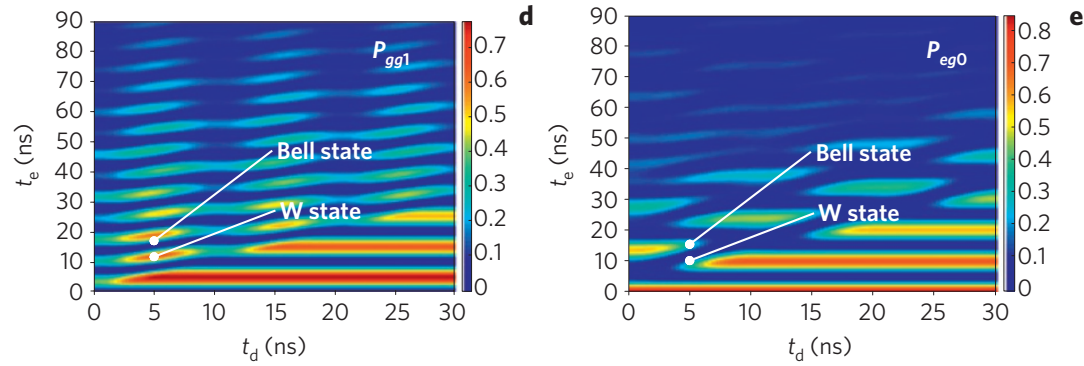
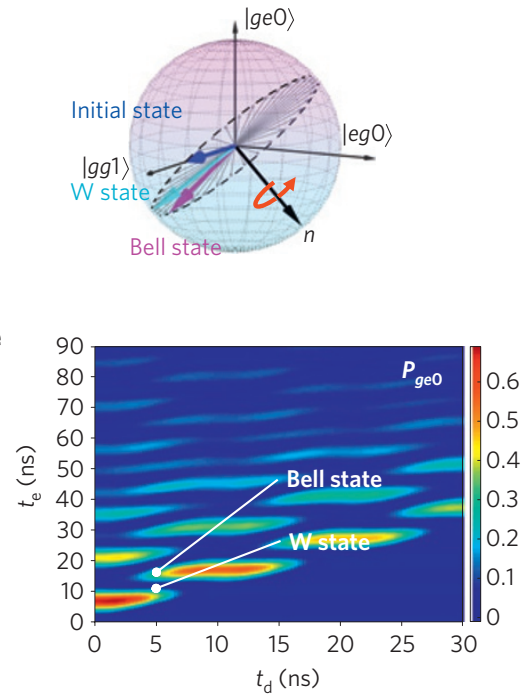

Figure 3 | Experimental protocol and theoretical predictions for generating generalized arbitrary single-photon tripartite evolutions. a, Pulse sequence. (1) A photon is inserted in the system by exciting qubit 1. (2) A shift pulse brings qubit 1 onto resonance with the cavity, producing vacuum Rabi oscillations. (3) A shift pulse brings qubit 2 onto resonance after the delay time $t_{\mathrm{d}}$, initiating tripartite interactions that evolve over a time period $t_{\mathrm{e}}-t_{\mathrm{d}}$. (4) Both qubits are measured simultaneously. $\mathbf{b}$, Tripartite sphere representation of the tripartite evolution for the initial state $|g g 1\rangle$ prepared during a delay time period $t_{\mathrm{d}}=\pi / \Omega_{0}$. The red arrow provides a visual cue to the circular trajectory of the tripartite vector. c, Predicted state occupation of one photon in the resonant cavity. $\mathbf{d}, \mathbf{e}$, Predicted joint state probabilities $P_{\mathrm{eg} 0}$ and $P_{\text {geo }}$ for measurement of qubit 1 and 2 as functions of both $t_{\mathrm{d}}$ and $t_{\mathrm{e}}$.

a
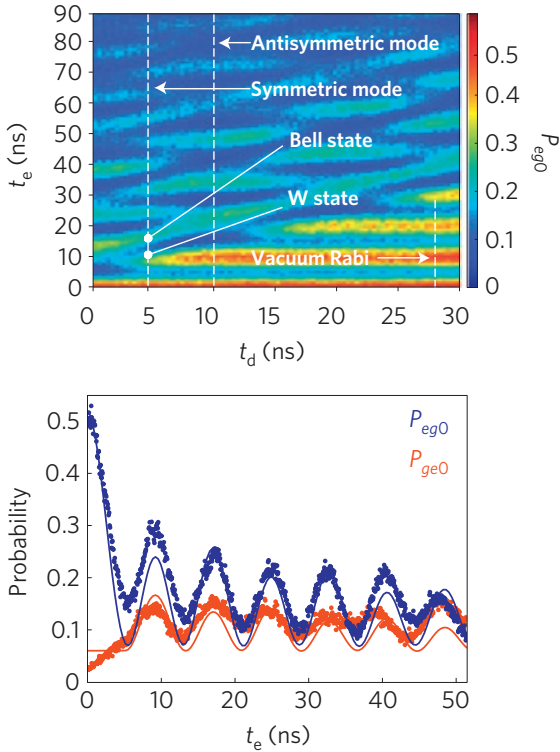
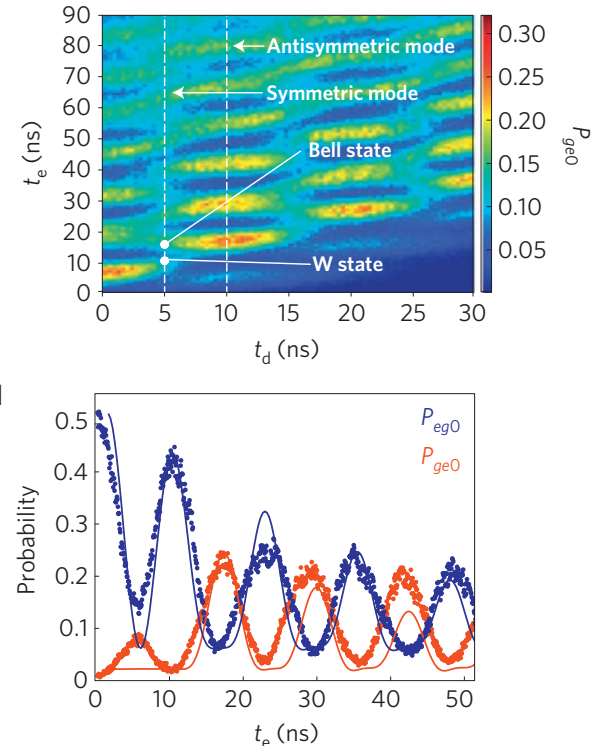

Figure 4 | Experimental demonstration of arbitrary tripartite interactions between both phase qubits and the cavity along with theoretical predictions. a,b, Measured joint state probabilities $P_{\mathrm{eg} 0}$ and $P_{\text {geo }}$ for measurement of qubit 1 and 2 as functions of both $t_{\mathrm{d}}$ and $t_{\mathrm{e}}$. c, Extracted curves (along dashed line in a) for the initial state $|g g 1\rangle$ producing a tripartite evolution of the symmetric mode, showing in-phase oscillations along with theoretical predictions (solid lines) from Fig. 3d,e. During this evolution, the system's entanglement continuously transforms starting from a pure state $|g g 1\rangle$. In the ideal case, the system evolves through W and Bell states. $\mathbf{d}$, Extracted curves (along dashed line in $\mathbf{b}$ ) for the initial state $\mid$ eg 0$\rangle$ producing a tripartite evolution of the antisymmetric mode, showing out-of-phase oscillations along with theoretical predictions (solid lines) from Fig. 3d,e.

of two-level system defects. This more than doubled the qubit visibility and provided the necessary 'clean' cavity region for observing tripartite interactions. However, it was not possible to carry out two-qubit state tomography over the required timescales owing to short relaxation times ${ }^{29}$ matched with the continued presence of two-level system defects that limited the qubit visibility $^{30}$. With further reductions in junction size, we can raise the single-qubit visibility to $90 \%$, allowing full tomographic characterization of both qubits ${ }^{13}$.
In the future, we intend to carry out correlated measurements and tomography of this tripartite system. This requires a fast, single-shot dispersive measurement and readout of the qubits to solve three difficulties. First, the tunnelling-based measurement of either qubit will populate the cavity with unwanted photons owing to a crosstalk process ${ }^{12}$. Second, a dispersive measurement will increase qubit visibility, ensuring clear tomography. Third, after measurement of the two qubits, subsequent qubit rotations will ensure proper state preparation for one of the qubits, making it 
ready for re-interaction with the cavity. In this way, we can reuse one of the qubits through state transfer ${ }^{14}$, to fully determine the cavity state ${ }^{19}$. Improvements are now underway to modify our slow switching-current superconducting quantum interference device readout to a fast, dispersive resonant readout.

Note added in proof: Recently, three-qubit entanglement has been demonstrated in two other superconducting systems $\mathrm{s}^{32,33}$.

\section{Methods}

The simulations in Figs $2 \mathrm{~d}-\mathrm{f}, 3 \mathrm{c}-\mathrm{e}$ and $4 \mathrm{c}, \mathrm{d}$ have been carried out by solving the master equation for this system. The master equation describing energy relaxation and dephasing within the Born and Markov approximations can be cast in the Linblad form

$$
\dot{\rho}=\mathcal{L} \rho=-i[H, \rho]+\sum_{k} \mathcal{D}\left[L_{k}\right] \rho
$$

where $\mathcal{L}$ is the Liouvillan and $\mathcal{D}$ are superoperators defined by

$$
\mathcal{D}[O] \rho \equiv O \rho O^{\dagger}-\frac{1}{2}\left(O^{\dagger} O \rho+\rho O^{\dagger} O\right)
$$

$L_{k}$ are jump operators describing the (environmental) measurement processes, corresponding to qubits and cavity relaxation and dephasing. For the two-qubit and cavity Hamiltonian under investigation, relaxation and dephasing rates are characterized by the respective spontaneous decay rates, $\gamma_{i}$ for qubits $i=1,2$ and $\kappa$ for the cavity, and dephasing rates, $\gamma_{i}^{\prime}$ and $\kappa^{\prime}$. The jump operators corresponding to energy relaxation are defined, in dimensionless units, by

$$
\begin{aligned}
L_{\mathrm{c}} & =\sqrt{2 \kappa} a \\
L_{i} & =\sqrt{\gamma_{i}} \sigma_{i}^{-}
\end{aligned}
$$

For the simulations reported here, the dephasing rates have been taken to be zero because the dominant effect of the environment is energy relaxation. The lifetimes of the two qubits and the cavity were measured individually and independently by excitation and subsequent decay using an exponential fit. We solve the time-dependent master equation using a Crank-Nicholson integration scheme. The time steps are taken to be much smaller than the characteristic interaction times from the qubit-cavity couplings (that is, $\mathrm{d} t \ll 1 / g \ll 1$ in the dimensionless case) and slower than the rate of change of any relevant dynamic systems parameters (typically $\mathrm{d} t \approx 0.001-0.0001$ were sufficient and little difference was seen beyond $\mathrm{d} t=0.01$ ) and the infinite harmonic oscillator states of the cavity are truncated at $N=7$, where $N$ needs to be more than the number of quanta in the systems (for the experiments considered typically little difference is seen above $N=3$ ). Similar numerical analysis has been used in a variety of quantum optical and circuit quantum electrodynamics settings ${ }^{31}$.

Using the measured contrast for each qubit, we determine a scale factor that is applied to the results of the simulation to match the visibility of the experiment. To incorporate the shift pulse into the simulations, we have assumed an exponential rise time of the form $1-\mathrm{e}^{-t / \tau_{\mathrm{RC}}}$ (as in a resistor-capacitor circuit) instead of a linear ramp, with $\tau_{\mathrm{RC}} \sim 10 \mathrm{~ns}$. This seems reasonable considering there is some stray capacitance in the feed lines and room-temperature measurements have shown this behaviour. In any case, the results of the simulation were relatively insensitive to the exact shape of the shift pulse and more sensitive to the timescales of the rise time with respect to characteristic interaction time of the systems. The asymmetry in Fig. $2 \mathrm{~d}-\mathrm{f}$ can be attributed to extra interference resulting from a residual finite detuning of qubit $1(\sim 0.3 g)$. Qubit 1 was detuned by the same amount in the simulations of Figs $3 \mathrm{c}-\mathrm{e}$ and $4 \mathrm{c}$,d.

\section{Received 2 December 2009; accepted 18 June 2010;} published online 1 August 2010

\section{References}

1. Gottesman, D. \& Chuang, I. L. Demonstrating the viability of universal quantum computation using teleportation and single-qubit operations. Nature 402, 390-393 (1999).

2. Karlsson, A. \& Bourennane, M. Quantum teleportation using three-particle entanglement. Phys. Rev. A 58, 4394-4400 (1998).

3. Briegel, H. J., Dür, W., Cirac, J. \& Zoller, P. Quantum repeaters: The role of imperfect local operations in quantum communication. Phys. Rev. Lett. 81, 5932-5935 (1998).

4. Hillery, M., Buzek, V. \& Berthiaume, A. Quantum secret sharing. Phys. Rev. A 59, 1829-1834 (1999).

5. Greenberger, D. M., Horne, M. A. \& Zeilinger, A. Multiparticle interferometry and the superposition principle. Phys. Today 46, 22-29 (1993).
6. Giovannetti, V., Lloyd, S. \& Maccone, L. Quantum-enhanced measurements: Beating the standard quantum limit. Science 306, 1330-1336 (2004).

7. Leibfried, D. et al. Creation of a six-atom Schrödinger cat state. Nature 438, 639-641 (2005).

8. Roos, C. F. et al. Control and measurement of three-qubit entangled states. Science 304, 1478-1482 (2004).

9. Pan, J-W., Bouwmeester, D., Daniell, M., Weinfurter, H. \& Zeilinger, A. Experimental test of quantum nonlocality in three-photon Greenberger-Horne-Zeilinger entanglement. Nature 403, 515-519 (2000).

10. Rauschenbeutel, A. et al. Step-by-step engineered multiparticle entanglement. Science 288, 2024-2028 (2000).

11. Pashkin, Y. A. et al. Quantum oscillations in two coupled charge qubits. Nature 421, 823-826 (2003)

12. McDermott, R. et al. Simultaneous state measurement of coupled Josephson phase qubits. Science 307, 1299-1302 (2005).

13. Steffen, M. et al. Measurement of the entanglement of two superconducting qubits via state tomography. Science 313, 1423-1425 (2006).

14. Sillanpää, M. A., Park, J. I. \& Simmonds, R. W. Coherent quantum state storage and transfer between two phase qubits via a resonant cavity. Nature 449, 438-442 (2007).

15. DiCarlo, L. et al. Demonstration of two-qubit algorithms with a superconducting quantum processor. Nature 460, 240-244 (2009).

16. Niskanen, A. O. et al. Quantum coherent tunable coupling of superconducting qubits. Science 316, 723-726 (2007).

17. Xu, H. et al. Spectroscopy of three-particle entanglement in a macroscopic superconducting circuit. Phys. Rev. Lett. 94, 027003 (2005).

18. Fink, J. M. et al. Dressed collective qubit states and the Tavis-Cummings model in circuit QED. Phys. Rev. Lett. 103, 083601 (2009).

19. Hofheinz, M. et al. Synthesizing arbitrary quantum states in a superconducting resonator. Nature 459, 546-549 (2009).

20. Hao, C-F., Li, J-C. \& Guo, G-C. Controlled dense coding using the Greenberger-Horne-Zeilinger state. Phys. Rev. A 63, 054301 (2001).

21. Borsten, L., Dahanayake, D., Duff, M. J., Ebrahim, H. \& Rubens, W. Wrapped branes as qubits. Phys. Rev. Lett. 100, 251602 (2008).

22. Dür, W., Vidal, G. \& Cirac, J. Three qubits can be entangled in two inequivalent ways. Phys. Rev. A 62, 062314 (2000).

23. Wei, L. F., Liu, Y-x. \& Nori, F. Greenberger-Horne-Zeilinger entanglement in superconducting circuits. Phys. Rev. Lett. 96, 246803 (2006).

24. Galiautdinov, A. \& Martinis, J. M. Maximally entangling tripartite protocols for Josephson phase qubits. Phys. Rev. A 78, 010305 (2008).

25. Kim, M. D. \& Cho, S. Y. Macroscopic Greenberger-Horne-Zeilinger and W states in flux qubits. Phys. Rev. B 77, 100508(R) (2008).

26. Simmonds, R. W. et al. Decoherence in Josephson phase qubits from junction resonators. Phys. Rev. Lett. 93, 077003 (2004).

27. Tavis, M. \& Cummings, F. W. Exact solution for an $n$-molecule-radiation-field Hamiltonian. Phys. Rev. 170, 379-384 (1968).

28. Simmonds, R. W. et al. Coherent interactions between phase qubits, cavities, and TLS defects. Quant. Inform. Process. 8, 117-131 (2009).

29. Martinis, J. M. et al. Decoherence in Josephson qubits from dielectric loss. Phys. Rev. Lett. 95, 210503 (2005).

30. Cooper, K. B. et al. Observation of quantum oscillations between a Josephson phase qubit and a microscopic resonator using fast readout. Phys. Rev. Lett. 93, 180401 (2004).

31. Blais, A. et al. Quantum-information processing with circuit quantum electrodynamics. Phys. Rev. A 75, 032329 (2007).

32. Neeley, M. et al. Generation of three-qubit entangled states using superconducting phase qubits. Preprint at http://arxiv.org/abs/1004.4246 (2010).

33. Dicarlo, L. et al. Preparation and measurement of three-qubit entanglement in a superconducting circuit. Preprint at http:/arxiv.org/abs/1004.4324 (2010)

\section{Acknowledgements}

This work was financially supported by NIST and in part by IARPA. M.A.S. was supported by the Academy of Finland and by the European Research Council (grant no. FP7-240387).

\section{Author contributions}

F.A., J.I.P., M.A.S. and R.W.S. developed the experiment. F.A. carried out the experiment. J.I.P. carried out the theoretical simulations. K.C. fabricated the device. J.D.W. developed the acquisition software. All authors discussed the results and contributed to the manuscript. F.A., J.I.P. and R.W.S. wrote the manuscript.

\section{Additional information}

The authors declare no competing financial interests. Reprints and permissions information is available online at http://npg.nature.com/reprintsandpermissions. Correspondence and requests for materials should be addressed to R.W.S. 